\title{
Hétérogénéité des récepteurs de l'endothéline
}

Le rôle majeur de l'endothélium vasculaire dans la régulation du tonus vasculaire a été établi par la découverte successive de facteurs vasodilatateurs (tels que l'endothelium-derived relaxing factor [EDRF] ou les prostacyclines) ou plus récemment de peptides vasoconstricteurs dont les plus puissants connus actuellement appartiennent à une famille de peptides de 21 acides aminés, les endothélines. Le premier peptide identifié, l'endothéline-1, a été mis en évidence en 1988 dans un surnageant de culture de cellules endothéliales d'aorte et provoque, lorsqu'il est injecté par voie intraveineuse, une hypertension prolongée [1]. Le clonage des gènes codant pour l'endothéline a révélé l'existence de deux autres formes de ce peptide, l'endothéline-2 et l'endothéline-3, également constituées de 21 acides aminés, mais dont la séquence diffère de celle de l'endothéline-1 par, respectivement, deux et six acides aminés [2]. De plus, les endothélines ont une structure similaire à celle de toxines de venin d'aspic, les sarafotoxines, qui se lient aux mêmes récepteurs [3]. Des trois formes d'endothélines, seule l'endothéline-1 est produite par les cellules endothéliales vasculaires, l'origine cellulaire de l'endothéline-2 et de l'endothéline-3 restant à déterminer. Toutefois, de nombreux auteurs ont suggéré une action locale des endothélines, puisque des ARN messagers codant pour ces trois peptides sont également détectés dans de nombreux tissus non vasculaires. En accord avec ces données, la présence de récepteurs des endothélines dans de nombreux tissus, corrélée à des effets biologiques divers, a permis de mettre en évidence des actions non vasculaires de ces peptides. Ainsi, des effets cardiaques, rénaux, des effets sur les glandes surrénales, sur l'intestin, l'utérus, le foie, un rôle mitogène $m / s n^{\circ} 4$, vol. 7, arril 91 et de neuropeptide ont été décrits (pour une revue, voir [4]).

L'existence de deux types de récepteurs de l'endothéline avait été suggérée par de nombreux auteurs sur une base biochimique. Mais ce sont des données de biologie moléculaire qui viennent d'établir indiscutablement l'existence de ces deux récepteurs. En effet, deux équipes japonaises ont décrit récemment le clonage et l'expression de deux types de récepteurs de l'endothéline qui possèdent sept domaines transmembranaires caractéristiques des récepteurs couplés aux G-protéines. L'ADN complémentaire cloné par l'équipe de Masaki [5], transfecté dans des cellules de mammifères COS dépourvues de récepteurs de l'endothéline, a conduit à l'expression d'un récepteur qui lie avec la même affinité les trois formes d'endothéline ainsi que la sarafotoxine, et est couplé à la phospholipase C. L'ARN messager de ce récepteur (ou récepteur $\mathrm{ET}_{\mathrm{B}}$ ) est présent dans un grand nombre de tissus non vasculaires mais n'est pas exprimé dans les cellules musculaires lisses d'aorte. En revanche, l'ADN complémentaire cloné par l'équipe de Nakanishi [6], transfecté dans les mêmes cellules COS, exprime un récepteur liant spécifiquement l'endothéline-1. L'ARN messager de ce récepteur (récepteur $\mathrm{ET}_{\mathrm{A}}$ ) a une taille inférieure à celle du récepteur $\mathrm{ET}_{\mathrm{B}}$, est également présent dans de nombreux tissus, mais son expression éventuelle dans les cellules musculaires lisses d'aorte reste à déterminer. Peut-on attribuer des fonctions différentes à ces deux récepteurs de l'endothéline? On sait que l'injection intraveineuse d'endothéline-1, d'endothéline-2 ou d'endothéline-3 chez le rat conduit tout d'abord à une diminution de la pression artérielle puis à une hypertension, l'endothéline- 3 possédant une activité hypotensive plus importante que celle de l'endothéline-1 [2]. L'endothéline-1 et l'endothéline-3 perfusées dans l'artère mésentérique provoquent toutes deux le relargage de substances vasodilatatrices (EDRF) ; cependant, l'endothéline-1 est un vasoconstricteur beaucoup plus actif que l'endothéline-3 [7]. Il est donc tentant de proposer que le récepteur spécifique de l'endothéline-1 (ET $\left.\mathrm{E}_{\mathrm{A}}\right)$ (pourrait-il s'agir du récepteur des cellules musculaires lisses d'aorte ?) relaye l'effet vasoconstricteur de l'endothéline alors que l'activation du récepteur non sélectif $\left(E T_{B}\right)$ conduirait à la production d'EDRF et ainsi à la vasodilatation. Il reste également à déterminer, dans des tissus non vasculaires exprimant les deux types de récepteurs, tels que le rein, le cœur ou les poumons, la réponse physiologique pouvant leur être spécifiquement associée.

S. $\mathbf{L}$.

1. Yanagisawa $\mathrm{M}$, Kurihata $\mathrm{H}, \mathrm{Kimura} \mathrm{S}$, $e t$ al. A novel potent vasoconstrictor peptide produced by vascular endothelial cells. Nature 1988 ; 322 : 411-5.

2. Inoue A, Yanagisawa M, Kimura S, et al. The human endothelin family : three structurally and pharmacologically distinct isopeptides predicted by three separate genes. Proc Natl Acad Sci USA 1989 ; 86: 2863-7.

3. Kloog Y, Sokolovsky M. Similarities in mode and sites of action of sarafotoxins and endothelins. Trends Pharmacol Sci 1989; 10 : 212-4.

4. Lotersztajn S. Les effets non vasculaires de l'endothéline. médecine/sciences $1991 ; 7: 78-9$. 5. Sakurai T, Yanagisawa M, Takuwa Y, et al. Cloning of a cDNA encoding a nonisopeptide-selective subtype of the endothelin receptor. Nature $1990 ; 348: 732-5$.

6. Arai $\mathrm{H}$, Hori $\mathrm{S}$, Aramori I, Ohkubo $\mathrm{H}$, Nakanishi S. Cloning and expression of a cDNA encoding an endothelin receptor. Nature $1990 ; 348: 730-2$.

7. Warner TD, De Nucci G, Vane JR. Eur $J$ Pharmacol 1989; 159 : 325-6.
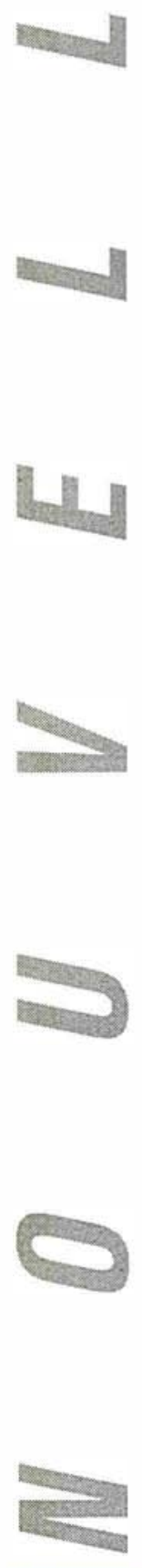